\title{
Assessing the Significance of Relationship Between Metrology Variables under Indeterminacy
}

\author{
M. Aslam*(1) \\ Department of Statistics, Faculty of Science, King Abdulaziz University, Jeddah 21551, Saudi Arabia
}

Received: 19 December 2020 / Accepted: 02 August 2021 / Published online: 27 September 2021

(C) Metrology Society of India 2021

\begin{abstract}
This paper introduces a Z-test for two correlation coefficients under neutrosophic statistics. The necessary steps to implement the proposed test are given. The statistic of the proposed test under indeterminacy introduced the first time. The application of the proposed test is given using temperature and wind speed data. From the analysis of the energy example, it is concluded that the proposed test is more efficient than the Z-test for two correlation coefficients under classical statistics in terms of the measure of indeterminacy, information, and flexibility. Based on the comparative study, it is recommended to apply the proposed test in the areas of energy and weather.
\end{abstract}

Keywords: Wind speed; Temperature; Energy; Weather; Neutrosophic statistics

\section{Introduction}

In practice, the practitioners, experimenters, and energy experts are interested to investigate the relationship between variables at different places and years. This objective can be obtained from the study of the correlation analysis. The Z-test for two correlation coefficients under classical statistics is quite helpful to the significance between two variables recorded at different places and years. The main aim of this test is to see either the relationship between a pair variable recorded at one place/year is significantly different from a pair of variables recorded at the second place or another year. This type of study will guide them to estimate/forecast the relationship between these variables for the next years. Kumar and Kumar [1] studied the relationship between metrological and COVID19 pandemic. Weaver and Wuensch [2], Lee [3], Chen [4], Giakoumis [5], Shan et al. [6] and Rezaee et al. [7] studied various applications of correlation analysis in a variety of fields.

Statistical analysis has been widely applied for analyzing the wind speed for forecasting purposes. The correlation analysis is also helpful in analyzing the wind speed and temperature relationship. For example, the energy experts or meteorologists may interest to see the

*Corresponding author, E-mail: aslam_ravian@hotmail.com relationship between the wind speed and temperature in the year 2019 and the wind speed and temperature in the year 2020. Bechrakis and Sparis [8] used the correlation to study the relationship between wind speed of various stations. Su et al. [9] studied the relation between wind speed and wind turbines. Shen et al. [10] presented the work on the correlation between energy variables. More applications of statistical method in forecasting and analysis of wind speed can be seen in Rahimiyan [11], Arias-Rosales and OsorioGómez [12], Katinas et al. [13], Min et al. [14], Barhmi et al. [15], and Ben et al. [16].

The Z-test for two correlation coefficients under classical statistics is workable under the assumption that the data follow the normal distribution and have all determined observations. In real life, the wind speed and temperature data are recorded in intervals. In such cases, when the data are intervals or there is uncertainty in parameters or observations, the statistical methods using fuzzy logic are applied. Damousis et al. [17] studied the relationship between energy-related variables using fuzzy logic. Some statistical tests developed using the fuzzy logic can be seen in Montenegro et al. [18], Petković [19], Grzegorzewski and Śpiewak [20], Sezer et al. [21] and Nie et al. [22].

The neutrosophic logic is said to be more efficient than the fuzzy logic as it gives additional information about the measure of indeterminacy, see Smarandache [23]. Smarandache and Khalid, [24] proved its efficiency over the fuzzy logic and interval-based analysis. Several applications of the neutrosophic logic can be read in Abdel- 
Basset et al. [25], Smarandache [26] and Nabeeh et al. [27]. Smarandache [28] introduced the neutrosophic statistics that gives efficient results when data are in the interval, imprecise and indeterminate. Chen et al. [29, 30] discussed the measure of indeterminacy evaluation for neutrosophic numbers. Aslam [31] presented the wind forecasting method under neutrosophic statistics. More details can be seen in Aslam [32] and Aslam [33].

A rich literature on wind speed analysis using correlation under classical statistics and fuzzy logic is available in the literature. The existing tests are unable to give information about the measure of indeterminacy. By exploring the literature and best of our knowledge, there is no work on Z-test for two correlation coefficients under neutrosophic statistics. In this paper, we will originally introduce a Z-test for two correlation coefficients under neutrosophic statistics. The statistic of the proposed test will introduce

$$
\begin{aligned}
& r_{1 N}=\frac{n_{N} \sum X_{1 N} Y_{1 N}-\sum X_{1 N} \sum Y_{1 N}}{\sqrt{\left\{n_{N} \sum X_{1 N}^{2}-\left(\sum X_{1 N}\right)^{2}\right\}\left\{n_{N} \sum Y_{1 N}^{2}-\left(\sum Y_{1 N}\right)^{2}\right\}}} ; n_{N} \\
& \in\left[n_{L}, n_{U}\right]
\end{aligned}
$$

The neutrosophic correlation for the second sample, say $r_{2 N} \in\left[r_{2 L}, r_{2 U}\right]$ can be defined as follows

$$
\begin{aligned}
r_{2 N} & =\frac{n_{N} \sum X_{2 N} Y_{2 N}-\sum X_{2 N} \sum Y_{2 N}}{\sqrt{\left\{n_{N} \sum X_{2 N}^{2}-\left(\sum X_{2 N}\right)^{2}\right\}\left\{n_{N} \sum Y_{2 N}^{2}-\left(\sum Y_{2 N}\right)^{2}\right\}}} ; n_{N} \\
& \in\left[n_{L}, n_{U}\right]
\end{aligned}
$$

The neutrosophic correlation, say $r_{N} \in\left[r_{1 N}, r_{2 N}\right]$ is defined as follows

$r_{N}=\left\{\frac{n_{N} \sum X_{1 N} Y_{1 N}-\sum X_{1 N} \sum Y_{1 N}}{\sqrt{\left\{n_{N} \sum X_{1 N}^{2}-\left(\sum X_{1 N}\right)^{2}\right\}\left\{n_{N} \sum Y_{1 N}^{2}-\left(\sum Y_{1 N}\right)^{2}\right\}}}, \frac{n_{N} \sum X_{2 N} Y_{2 N}-\sum X_{2 N} \sum Y_{2 N}}{\sqrt{\left\{n_{N} \sum X_{2 N}^{2}-\left(\sum X_{2 N}\right)^{2}\right\}\left\{n_{N} \sum Y_{2 N}^{2}-\left(\sum Y_{2 N}\right)^{2}\right\}}}\right\}$

under indeterminacy. The application of the proposed test will be given on the energy data with the expectation that it will be efficient and flexible to apply under uncertainty. It is expected that the proposed test will be helpful for studying the relationship between wind speed and temperature at various places, stations and years.

\section{Preliminaries}

Suppose that $X_{i 1 N}=X_{i 1 L}+X_{i 1 U} I_{1 N}\left(i=1,2,3, \ldots, n_{N}\right)$; $I_{1 x N} \in\left[I_{1 x L}, I_{1 U}\right] \quad$ and $\quad Y_{i 1 N}=Y_{i 1 L}+Y_{i 1 U} I_{1 N}(i=1,2$, $\left.3, \ldots, n_{N}\right) ; I_{1 y N} \in\left[I_{1 y L}, I_{1 y U}\right]$ be a pair of neutrosophic random of the first sample of sample size $n_{N} \in\left[n_{L}, n_{U}\right]$. Let $X_{i 2 N}=X_{i 2 L}+X_{i 2 U} I_{2 N}\left(i=1,2,3, \ldots, n_{N}\right) ; I_{2 x N} \in\left[I_{2 x L}, I_{2 U}\right]$ and $\quad X_{i 2 N}=Y_{i 2 L}+Y_{i 2 U} I_{2 N}\left(i=1,2,3, \ldots, n_{N}\right) ; I_{2 y N} \in$ $\left[I_{2 y L}, I_{2 y U}\right]$ be a pair of neutrosophic random of the second sample, where $I_{1 x N} \in\left[I_{1 x L}, I_{1 U}\right]$ and $I_{2 y N} \in\left[I_{2 y L}, I_{2 y U}\right]$ present the corresponding measure of indeterminacy. Note that $X_{i 1 L}, Y_{i 1 L}, X_{i 2 L}$ and $Y_{i 2 L}$ present the determined observations and $X_{i 1 U} I_{1 N}, Y_{i 1 U} I_{1 N}, X_{i 2 L}$ and $Y_{i 2 L}$ are indeterminate part of neutrosophic forms. Based on this information, the neutrosophic correlation for the first sample, say $r_{1 N} \in$ $\left[r_{1 L}, r_{1 U}\right]$ can be defined as follows
The neutrosophic form $r_{N} \in\left[r_{1 N}, r_{2 N}\right]$ can be written as

$$
\begin{aligned}
r_{N} & =r_{1 N}+r_{2 N} I_{r N} ; I_{r N} \in\left[I_{r L}, I_{r U}\right], r_{1 N} \in\left[r_{1 L}, r_{1 U}\right], r_{2 N} \\
& \in\left[r_{2 L}, r_{2 U}\right]
\end{aligned}
$$

In Eq. (4), the first part $r_{1 N} \in\left[r_{1 L}, r_{1 U}\right]$ denotes the correlation under classical statistics and $r_{2 N} I_{r N}$ denotes the indeterminate part, where $I_{r N} \in\left[I_{r L}, I_{r U}\right]$ presents the indeterminacy associated with $r_{N} \in\left[r_{1 N}, r_{2 N}\right]$. The neutrosophic form reduces to correlation under classical statistics if $I_{r L}=0$.

\section{Design of the Proposed Test}

The existing Z-test for two correlation coefficients has been applied widely for testing the significant difference between correlation coefficients when the observations in each pair of two samples are precise, exact and determined. The use of the existing test may mislead when the samples have intervals, inexact and indeterminate observations. In this section, the design of the proposed Z-test for two correlation coefficients under neutrosophic statistics will be presented. It is assumed that the two samples are drawn from the neutrosophic normal distributions and the relationship between the neutrosophic independent variables 
and neutrosophic dependent variables is linear. Suppose that $\rho_{1 N}$ and $\rho_{2 N}$ are the corresponding neutrosophic population correlations, see Kanji [34] and Smarandache [28]. The Z-test of a correlation coefficient for the first sample under neutrosophic statistics is calculated as follows

$$
\begin{aligned}
Z_{1 N} & =\frac{1}{2} \log _{e}\left(\frac{1+r_{1 N}}{1-r_{1 N}}\right)=1.1513 \log _{10}\left(\frac{1+r_{1 N}}{1-r_{1 N}}\right) ; r_{1 N} \\
& \in\left[r_{1 L}, r_{1 U}\right]
\end{aligned}
$$

In neutrosophic form, the values of $Z_{1 N} \in\left[Z_{1 L}, Z_{1 U}\right]$ can be written as

$Z_{1 N}=Z_{1 L}+Z_{1 U} I_{z 1 N} ; I_{z 1 N} \in\left[I_{z 1 L}, I_{z 1 U}\right]$

The Z-test of a correlation coefficient for the second sample under neutrosophic statistics is calculated as follows

$$
\begin{aligned}
Z_{2 N} & =\frac{1}{2} \log _{e}\left(\frac{1+r_{2 N}}{1-r_{2 N}}\right)=1.1513 \log _{10}\left(\frac{1+r_{2 N}}{1-r_{2 N}}\right) ; r_{2 N} \\
& \in\left[r_{2 L}, r_{2 U}\right]
\end{aligned}
$$

In neutrosophic form, the values of $Z_{2 N} \in\left[Z_{2 L}, Z_{2 U}\right]$ can be written as

$Z_{2 N}=Z_{2 L}+Z_{2 U} I_{z 2 N} ; I_{z 2 N} \in\left[I_{z 2 L}, I_{z 2 U}\right]$

The neutrosophic mean of $Z_{1 N} \in\left[Z_{1 L}, Z_{1 U}\right]$ and $Z_{2 N} \in$ $\left[Z_{2 L}, Z_{2 U}\right]$ are given by

$\mu_{Z_{1 N}}=\frac{1}{2} \log _{e}\left(\frac{1+\rho_{1 N}}{1-\rho_{1 N}}\right)=1.1513 \log _{10}\left(\frac{1+\rho_{1 N}}{1-\rho_{1 N}}\right)$

$\mu_{Z_{2 N}}=\frac{1}{2} \log _{e}\left(\frac{1+\rho_{2 N}}{1-\rho_{2 N}}\right)=1.1513 \log _{10}\left(\frac{1+\rho_{2 N}}{1-\rho_{2 N}}\right)$

The neutrosophic variance of $Z_{1 N} \in\left[Z_{1 L}, Z_{1 U}\right]$ and $Z_{2 N} \in\left[Z_{2 L}, Z_{2 U}\right]$ are given by

$\sigma_{Z_{1 N}}=\frac{1}{\sqrt{\left(n_{1 N}\right)-3}}$

$\sigma_{Z_{2 N}}=\frac{1}{\sqrt{\left(n_{2 N}\right)-3}}$

The test statistic, say $Z_{N} \in\left[Z_{L}, Z_{U}\right]$ under neutrosophic statistics is given by

$Z_{N}=\frac{\left(Z_{1 N}-Z_{2 N}\right)-\left(\mu_{Z_{1 N}}-\mu_{Z_{2 N}}\right)}{\sigma_{N}} ; Z_{N} \in\left[Z_{L}, Z_{U}\right]$,

where $\sigma_{N} \in\left[\sigma_{L}, \sigma_{U}\right]$ is defined by

$\sigma_{N}=\sqrt{\sigma_{Z_{1 N}}^{2}+\sigma_{Z_{2 N}}^{2}}$

In neutrosophic form, the statistic $Z_{N} \in\left[Z_{L}, Z_{U}\right]$ can be written as
$Z_{N}=Z_{L}+Z_{U} I_{Z N} ; I_{Z N} \in\left[I_{Z L}, I_{Z U}\right]$

Note here that the statistic $Z_{N} \in\left[Z_{L}, Z_{U}\right]$ reduces to statistic under classical statistics when $I_{Z L}=0$ and $I_{Z N} \in$ $\left[I_{Z L}, I_{Z U}\right]$ is indeterminacy interval associated with $Z_{N} \in\left[Z_{L}, Z_{U}\right]$.

The proposed test can be implemented as follows

Step 1: State the null hypothesis that $H_{0 N}: \rho_{1 N}=\rho_{2 N}$ vs. the alternative hypothesis $H_{1 N}: \rho_{1 N} \neq \rho_{2 N}$.

Step 2: State the level of significance $\alpha$.

Step 3: Compute the values of the test statistic $Z_{N} \in$ $\left[Z_{L}, Z_{U}\right]$ using the neutrosophic sample information.

Step 4: Select the critical value from the Z-table corresponding to $\alpha$ and decide about the rejection region according to $H_{1 N}$.

Step 5: Do not reject $H_{0 N}: \rho_{1 N}=\rho_{2 N}$ is the calculated value of $Z_{N} \in\left[Z_{L}, Z_{U}\right]$ falls within the acceptance region.

\section{Application Using Energy Data}

In this section, the application of the proposed test is given on the weather data. For the study, the two important weather variables, namely temperature and wind speed are selected. The purpose of the application of the proposed test is to show the significant relationship between the temperature and wind speed at various time periods. The minimum and maximum values of two variables for the month of January are recoded for Lahore, Pakistan, and reported in Table 1 for the years 2019 and 2020. The energy experts are interested to see either the relation between temperature and wind speed for the year 2019, and the year 2020 is significant or not. As the data are recorded in indeterminate intervals, therefore the use of the existing test under classical statistics is not appropriate or may mislead the energy experts. In this situation, the proposed test can be applied to see either the correlation between temperature and wind for the two years is significant or not. Let $r_{1 N} \in\left[r_{1 L}, r_{1 U}\right]$ be the correlation between wind speed, say $X_{1 N}$ and temperature, say $Y_{1 N}$ for the year 2019. Let $r_{2 N} \in\left[r_{2 L}, r_{2 U}\right]$ be the correlation between wind speed, say $X_{2 N}$ and temperature, say $Y_{2 N}$ for the year 2020. The neutrosophic correlation $r_{1 N} \in\left[r_{1 L}, r_{1 U}\right]$ and $r_{2 N} \in$ $\left[r_{2 L}, r_{2 U}\right]$ are computed as follows

$r_{1 N} \in[-0.0763,-0.3190] ; n_{1 N} \in[31,31] \quad$ and $\quad r_{2 N} \in$ $[0.2804,0.1290] ; n_{2 N} \in[31,31]$.

The neutrosophic form $r_{N} \in\left[r_{1 N}, r_{2 N}\right]$ can be written as

$$
\begin{aligned}
r_{N}= & {\left[-0.0763+(-0.3190) I_{1 r N}\right] } \\
& +[0.2804-0.1290] I_{2 r N} ; I_{r N} \\
\in & {[0,0 ; 0.76,1.17] }
\end{aligned}
$$

The Z-test of a correlation coefficient for the first sample is computed as follows 
Table 1 Temperature and wind speed data

\begin{tabular}{|c|c|c|c|c|c|c|c|c|}
\hline \multirow[t]{3}{*}{ January } & \multicolumn{4}{|l|}{2019} & \multicolumn{4}{|l|}{2020} \\
\hline & \multicolumn{2}{|c|}{ Temperature $\left({ }^{\circ} \mathrm{F}\right)$} & \multicolumn{2}{|c|}{ Wind speed (mph) } & \multicolumn{2}{|c|}{ Temperature $\left({ }^{\circ} \mathrm{F}\right)$} & \multicolumn{2}{|c|}{ Wind speed (mph) } \\
\hline & Minimum & Maximum & Minimum & Maximum & Minimum & Maximum & Minimum & Maximum \\
\hline 1 & 43 & 63 & 0 & 48 & 36 & 52 & 0 & 6 \\
\hline 2 & 39 & 64 & 0 & 6 & 39 & 63 & 0 & 7 \\
\hline 3 & 41 & 66 & 0 & 10 & 43 & 68 & 0 & 6 \\
\hline 4 & 39 & 66 & 0 & 7 & 41 & 61 & 0 & 3 \\
\hline 5 & 46 & 59 & 0 & 13 & 43 & 59 & 0 & 7 \\
\hline 6 & 41 & 63 & 0 & 9 & 46 & 52 & 0 & 12 \\
\hline 7 & 39 & 63 & 0 & 29 & 48 & 52 & 0 & 7 \\
\hline 8 & 43 & 66 & 0 & 12 & 45 & 54 & 0 & 7 \\
\hline 9 & 43 & 70 & 0 & 12 & 43 & 59 & 0 & 8 \\
\hline 10 & 43 & 72 & 0 & 9 & 39 & 63 & 0 & 12 \\
\hline 11 & 48 & 66 & 0 & 7 & 45 & 64 & 2 & 9 \\
\hline 12 & 45 & 63 & 0 & 14 & 52 & 66 & 0 & 14 \\
\hline 13 & 45 & 66 & 0 & 13 & 46 & 54 & 0 & 21 \\
\hline 14 & 41 & 66 & 3 & 12 & 45 & 55 & 0 & 8 \\
\hline 15 & 41 & 70 & 0 & 8 & 41 & 57 & 0 & 6 \\
\hline 16 & 43 & 63 & 0 & 6 & 41 & 61 & 0 & 9 \\
\hline 17 & 36 & 66 & 0 & 9 & 39 & 61 & 0 & 5 \\
\hline 18 & 41 & 68 & 0 & 8 & 39 & 59 & 0 & 6 \\
\hline 19 & 43 & 70 & 0 & 8 & 43 & 63 & 0 & 10 \\
\hline 20 & 50 & 70 & 0 & 21 & 45 & 66 & 0 & 9 \\
\hline 21 & 48 & 59 & 0 & 13 & 50 & 63 & 3 & 10 \\
\hline 22 & 48 & 57 & 0 & 46 & 46 & 64 & 0 & 100 \\
\hline 23 & 41 & 61 & 0 & 8 & 45 & 64 & 0 & 15 \\
\hline 24 & 41 & 61 & 0 & 8 & 43 & 68 & 0 & 12 \\
\hline 25 & 39 & 64 & 0 & 9 & 48 & 66 & 0 & 9 \\
\hline 26 & 39 & 63 & 0 & 9 & 43 & 70 & 0 & 12 \\
\hline 27 & 41 & 64 & 0 & 12 & 46 & 66 & 0 & 12 \\
\hline 28 & 39 & 63 & 0 & 10 & 50 & 55 & 0 & 9 \\
\hline 29 & 37 & 66 & 0 & 8 & 48 & 61 & 0 & 9 \\
\hline 30 & 45 & 61 & 0 & 14 & 45 & 61 & 0 & 9 \\
\hline 31 & 48 & 55 & 0 & 15 & 43 & 66 & 0 & 8 \\
\hline
\end{tabular}

$$
\begin{aligned}
Z_{1 N} & =1.1513 \log _{10}\left(\frac{1+[-0.0763,-0.3190]}{1-[-0.0763,-0.3190]}\right) \\
& =[-0.0765,-0.3306]
\end{aligned}
$$

In neutrosophic form, the values of $Z_{1 N} \in\left[Z_{1 L}, Z_{1 U}\right]$ given as

$Z_{1 N}=-0.0765+(-0.3306) I_{z 1 N} ; I_{z 1 N} \in[0,0.7686]$

The Z-test of a correlation coefficient for the second sample under neutrosophic statistics is computed as follows

$$
\begin{aligned}
Z_{2 N} & =1.1513 \log _{10}\left(\frac{1+[0.2804,0.1290]}{1-[0.2804,0.1290]}\right) \\
& =[0.2881,0.1298]
\end{aligned}
$$

In neutrosophic form, the values of $Z_{2 N} \in\left[Z_{2 L}, Z_{2 U}\right]$ can be written as

$Z_{2 N}=0.2881-0.1298 I_{z 2 N} ; I_{z 2 N} \in[0,1.2196]$

The test statistic, say $Z_{N} \in\left[Z_{L}, Z_{U}\right]$ under neutrosophic statistics is computed as

$$
Z_{N}=\frac{[-0.0765,-0.3306]}{\sigma_{N}} ; \quad \sigma_{N} \in[0.2672,0.2672] \quad \text { and }
$$
$\left|Z_{N}\right| \in[1.3642,1.7226]$. by

In neutrosophic form, the statistic $Z_{N} \in\left[Z_{L}, Z_{U}\right]$ is given 
$Z_{N}=1.3642+1.7226 I_{Z N} ; I_{Z N} \in[0,0.2081]$

The proposed test for the real data sets is stated as follows

Step 1: State the null hypothesis that $H_{0 N}: \rho_{1 N}=\rho_{2 N}$ vs. the alternative hypothesis $H_{1 N}: \rho_{1 N} \neq \rho_{2 N}$.

Step 2: State the level of significance $\alpha=0.05$.

Step 3: Compute the values of the test statistic $\left|Z_{N}\right| \in$ $[1.3642,1.7226]$ using the neutrosophic sample information.

Step 4: The critical value from the Z-table is 1.96 corresponding to $\alpha=0.05$ and $H_{1 N}: \rho_{1 N} \neq \rho_{2 N}$.

Step 5: Do not reject $H_{0 N}: \rho_{1 N}=\rho_{2 N}$ as $\left|Z_{N}\right|<1.96$

From the proposed test, it can be concluded that the relationship between the temperature and wind speed of January 2019 and January 2020 is insignificant. Similarly, the relationship between variables can be studied for other months of the years 2019 and 2020.

\section{Comparative Study}

As discussed earlier, the proposed test is a generalization of the test under classical statistics. The proposed test reduces to the existing test when all observations in the data are exact, determined and certain. In this section, the comparison of the proposed test is given over the existing test in terms of the measure of indeterminacy, flexibility and information. For the comparison purpose, the neutrosophic form of the statistic $Z_{N} \in\left[Z_{L}, Z_{U}\right]$ is considered only. The other neutrosophic quantities can be explained in the same manner. The neutrosophic form of $Z_{N} \in\left[Z_{L}, Z_{U}\right]$ is $Z_{N}=1.3642+1.7226 I_{Z N} ; I_{Z N} \in[0,0.2081]$. Note here that this neutrosophic form reduces to statistic under classical statistics when $I_{Z L}=0$. Therefore, the first part of the neutrosophic form presents the value of test statistic under classical statistics. Similarly, the second part $1.7226 I_{Z N}$ shows the indeterminate part of the neutrosophic form. In addition, the measure of indeterminacy associated with this test is 0.2081 . According to the proposed test, the values of the statistic of statistic $Z_{N} \in\left[Z_{L}, Z_{U}\right]$ are flexible and lie in the indeterminate interval that is $\left|Z_{N}\right| \in[1.3642,1.7226]$. According to the proposed test, under an uncertain environment, the value of $Z_{N} \in\left[Z_{L}, Z_{U}\right]$ can be expected from 1.3642 to 1.7226 . This range differentiates the proposed test from the existing test under classical statistics which gives the determined value which is not appropriate in uncertainty. Another aspect of the proposed test is that it gives more information about the testing process under indeterminacy. The proposed test gives additional information about the testing procedure which is the measure of indeterminacy. For the energy example, for testing $H_{0 N}: \rho_{1 N}=\rho_{2 N}$, the probability that $H_{0 N}: \rho_{1 N}=\rho_{2 N}$ will be accepted is 0.95 , the change of rejecting it when it is true is 0.05 and change of uncertainty about $H_{0 N}: \rho_{1 N}=$ $\rho_{2 N}$ is 0.2081 . For fuzzy statistics, there are lower value of the interval (measure of truth) and the upper value of the interval (measure of falseness). It means that $Z_{N} \in\left[Z_{L}, Z_{U}\right]$ can be from 1.3642 to 1.7226 . The analysis based on fuzzy statistics does not give information about the parameter "measure of indeterminacy." From this study, it is clear that the proposed test is flexible, informative and reasonable to apply for testing $H_{0 N}: \rho_{1 N}=\rho_{2 N}$ under uncertainty. From the study, it is concluded that the proposed test under neutrosophic statistics is better than the test under classical and fuzzy statistics in terms of information and flexibility.

\section{Concluding Remarks}

This paper introduced a Z-test for two correlation coefficients under neutrosophic statistics. The necessary steps to implement the proposed test were given. The statistic of the proposed test under indeterminacy was introduced the first time. The application of the proposed test was given using temperature and wind speed data. The proposed test was the extension of the existing test under classical statistics. The application of the proposed test on the energy data showed it is efficient in measure of indeterminacy, flexibility and information. The proposed test can be applied for ocean big data as future research. The efficiency of the proposed test using some distributions can be considered a fruitful area of future research.

Acknowledgements We are thankful to the editor and reviewers for their valuable suggestions to improve the quality of the paper.

Data availability The data are given in the paper.

\section{Declarations}

Conflict of interest The author declares no conflict of interest.

\section{References}

[1] G. Kumar and R.R. Kumar, A correlation study between meteorological parameters and COVID-19 pandemic in Mumbai, India, Diabetes Metab. Syndr. Clin. Res. Rev., 14(6) (2020) 1735-1742.

[2] B. Weaver and K.L. Wuensch, SPSS and SAS programs for comparing Pearson correlations and OLS regression coefficients, Behav. Res. Methods, 45(3) (2013) 880-895.

[3] M.-Y. Lee, The effect of nonzero autocorrelation coefficients on the distributions of Durbin-Watson test estimator: three autoregressive models, Expert J. Econ., 2(3) (2014), 85-99.

[4] Y. Chen, Spatial autocorrelation approaches to testing residuals from least squares regression, PLoS One, 11(1) (2016) e0146865. 
[5] E.G. Giakoumis, Analysis of 22 vegetable oils' physico-chemical properties and fatty acid composition on a statistical basis, and correlation with the degree of unsaturation, Renew. Energy, 126 (2018) 403-419.

[6] G. Shan, H. Zhang and T. Jiang, Correlation coefficients for a study with repeated measures. Comput. Math. Methods Med., 2020 (2020).

[7] Z. Rezaee, S. Aliabadi, A. Dorestani and N.J. Rezaee, Application of time series models in business research: correlation, association, causation, Sustainability, 12(12) (2020) 4833.

[8] D.A. Bechrakis and P.D. Sparis, Correlation of wind speed between neighboring measuring stations, IEEE Trans. Energy Convers., 19(2) (2004) 400-406.

[9] C. Su, Q. Jin and Y. Fu, Correlation analysis for wind speed and failure rate of wind turbines using time series approach, J. Renew. Sustain. Energy, 4(3) (2012) 032301.

[10] X. Shen, C. Zhou and X. Fu, Study of time and meteorological characteristics of wind speed correlation in flat terrains based on operation data, Energies, 11(1) (2018) 219.

[11] M. Rahimiyan, A statistical cognitive model to assess impact of spatially correlated wind production on market behaviors, Appl. Energy, 122 (2014) 62-72.

[12] A. Arias-Rosales and G. Osorio-Gómez, Wind turbine selection method based on the statistical analysis of nominal specifications for estimating the cost of energy, Appl. Energy, 228 (2018) 980-998.

[13] V. Katinas, G. Gecevicius, and M. Marciukaitis, An investigation of wind power density distribution at location with low and high wind speeds using statistical model, Appl. Energy, 218 (2018) 442-451.

[14] Y. Min, Y. Chen and H. Yang, A statistical modeling approach on the performance prediction of indirect evaporative cooling energy recovery systems, Appl. Energy, 255 (2019) 113832.

[15] S. Barhmi, O. Elfatni and I. Belhaj, Forecasting of wind speed using multiple linear regression and artificial neural networks. Energy Syst., (2019) 1-12.

[16] Y. Ben, Y. Mei, Y. Chen, T. Hu and D. Zhu, Inter-and intraannual wind speed variabilities in wide valley regions of the middle reaches of the Yarlung Tsangpo River, China. Sci. Rep., 10(1) (2020) 1-20.

[17] I.G. Damousis, M.C. Alexiadis, J.B. Theocharis and P.S. Dokopoulos, A fuzzy model for wind speed prediction and power generation in wind parks using spatial correlation. IEEE Trans. Energy Convers., 19(2) (2004) 352-361.

[18] M. Montenegro, M.R. Casals, M.A. Lubiano and M.A. Gil, Two-sample hypothesis tests of means of a fuzzy random variable, Inf. Sci., 133(1-2) (2001) 89-100.

[19] D. Petković, Adaptive neuro-fuzzy approach for estimation of wind speed distribution, Int. J. Electr. Power Energy Syst., 73 (2015) 389-392.
[20] P. Grzegorzewski and M. Śpiewak, The sign test and the signedrank test for interval-valued data, Int. J. Intell. Syst., 34(9) (2019) 2122-2150.

[21] O.B. Sezer, M.U. Gudelek and A.M. Ozbayoglu, Financial time series forecasting with deep learning: a systematic literature review: 2005-2019, Appl. Soft Comput., 90 (2020) 106181.

[22] Y. Nie, H. Bo, W. Zhang and H. Zhang, Research on hybrid wind speed prediction system based on artificial intelligence and double prediction scheme, Complexity, 2020 (2020).

[23] F. Smarandache, Neutrosophy. Neutrosophic probability, set, and logic, ProQuest information \& learning, Ann Arbor, Michigan, USA, 105 (1998) 118-123.

[24] F. Smarandache and H.E. Khalid, Neutrosophic precalculus and neutrosophic calculus. Infinite Study (2015).

[25] M. Abdel-Basset, N.A. Nabeeh, H.A. El-Ghareeb and A. Aboelfetouh, Utilising neutrosophic theory to solve transition difficulties of IoT-based enterprises, Enterpr. Inf. Syst., (2019) $1-21$.

[26] F. Smarandache, Neutrosophic set is a generalization of intuitionistic fuzzy set, inconsistent intuitionistic fuzzy set (picture fuzzy set, ternary fuzzy set), pythagorean fuzzy set, spherical fuzzy set, and q-rung orthopair fuzzy set, while neutrosophication is a generalization of regret theory, grey system theory, and three-ways decision (revisited), J. New Theory 29 (2019) 1-31.

[27] N.A. Nabeeh, F. Smarandache, M. Abdel-Basset, H.A. ElGhareeb and A. Aboelfetouh, An integrated neutrosophic-topsis approach and its application to personnel selection: a new trend in brain processing and analysis, IEEE Access, 7 (2019) 29734-29744.

[28] F. Smarandache, Introduction to neutrosophic statistics. Infinite Study (2014)

[29] J. Chen, J. Ye and S. Du, Scale effect and anisotropy analyzed for neutrosophic numbers of rock joint roughness coefficient based on neutrosophic statistics. Symmetry, 9(10) (2017) 208.

[30] J. Chen, J. Ye, S. Du and R. Yong, Expressions of rock joint roughness coefficient using neutrosophic interval statistical numbers, Symmetry, 9(7) (2017) 123.

[31] M. Aslam, Forecasting of the wind speed under uncertainty, Sci. Rep., 10(1) (2020) 1-7.

[32] M. Aslam, Design of the Bartlett and Hartley tests for homogeneity of variances under indeterminacy environment, J. Taibah Univ. Sci, 14(1) (2020) 6-10.

[33] M. Aslam, On detecting outliers in complex data using Dixon's test under neutrosophic statistics, J. King Saud Univ. Sci. (2020).

[34] G.K. Kanji, 100 statistical tests. Sage (2006).

Publisher's Note Springer Nature remains neutral with regard to jurisdictional claims in published maps and institutional affiliations. 